\title{
BCS-BEC crossover with unequal-mass fermions
}

\author{
Roberto B. Diener* and Mohit Randeria \\ Department of Physics, The Ohio State University, Columbus, Ohio 43210, USA
}

(Received 23 June 2009; published 15 March 2010)

\begin{abstract}
We investigate the crossover from BCS pairing to molecular Bose-Einstein condensation (BEC) in an atomic gas with two fermion species with masses $m_{\uparrow} \neq m_{\downarrow}$ tuned through a Feshbach resonance. We present results for the $T=0$ equation of state as a function of the scattering length including the effects of Gaussian fluctuations about the mean field ground state. We compute the ground state energy as a function of $m_{\uparrow} / m_{\downarrow}$ at unitarity and find excellent agreement with the quantum Monte Carlo result for $m_{\uparrow} / m_{\downarrow}=6.67$ for a ${ }^{40} \mathrm{~K}-{ }^{6} \mathrm{Li}$ mixture. We show that the dimer scattering length in the BEC limit as a function of $m_{\uparrow} / m_{\downarrow}$ compares well with the exact four-body results of Petrov et al. [J. Phys. B 38, S645 (2005)]. We also derive the condition for trapping frequencies to obtain an unpolarized gas in a harmonic trap.
\end{abstract}

DOI: $10.1103 /$ PhysRevA.81.033608

PACS number(s): 67.85.-d, 03.75.Ss, 05.30.Fk, 05.30.Pr

The crossover [1-3] of collective BCS pairing to the Bose-Einstein condensation (BEC) of tightly bound molecules is of great interest in diverse areas of physics ranging from condensed matter to nuclear and high-energy physics. The unitary scattering regime which lies in the middle of the crossover in three dimensions permits us to gain a deeper understanding of superfluidity in a strongly interacting Fermi system. Recent advances in atomic cooling and trapping and the use of a Feshbach resonance to tune the interactions through unitarity have led to the experimental realization [4] of the BCS-BEC crossover in ultracold atomic gases.

In this paper we turn to the question of pairing and superfluidity in a mixture of two species of fermions with different masses. There are several motivations for undertaking such an investigation. First, from an experimental point of view, interspecies Feshbach resonances have already been observed in mixtures of fermionic ${ }^{6} \mathrm{Li}$ and ${ }^{40} \mathrm{~K}$ [5]. Moreover, for a dilute Fermi gas in an optical lattice, the effective masses of the two species can be independently tuned, allowing for a continuous variation of the mass ratio. This provides a whole new system in which strong interactions in the BCS-BEC crossover can be studied. Second, in high-energy physics examples, like color superconductivity, pairing naturally occurs between fermions with different masses [6]. Third, from a general many-body physics point of view it is interesting to ask if the unequal "spin" $\uparrow$ and $\downarrow$ mass, which breaks "time-reversal" symmetry in condensed matter systems, affects the pairing of $|\mathbf{k}, \uparrow\rangle$ and $|-\mathbf{k}, \downarrow\rangle$ states.

Here we present results for the $T=0$ equation of state for an unpolarized system for arbitrary scattering length and mass ratio. We generalize to the case of unequal masses a functional integral formulation that goes beyond the mean field (MF) theory results reported recently [8] and incorporates the effects of quantum fluctuations [7]. Specifically, our approach includes the energy of the zero-point motion of the collective mode, the Goldstone mode in the broken symmetry superfluid state, and the effects of virtual scattering of two-particle excitations which are clearly missing from the MF description. These physical processes, arising from particle-particle

\footnotetext{
*rdiener@mps.ohio-state.edu
}

channel contributions, are expected to dominate in the low density limit even though there is no small parameter.

Our results (Figs. 1-3) are significantly different from MF theory, and also from those of the $1 / \mathcal{N}$ approximation [9], but are in very good agreement with the few known exact results. At unitarity, our results compare well (Fig. 2) with quantum Monte Carlo results that are available for two values of the mass ratio: equal masses [10] and Li-K mixtures [11]. In the BEC limit we find that our dimer scattering length as a function of the mass ratio (Fig. 3) is in very good agreement with the exact four-body results [12]. In the BEC regime we also find approximately $90 \%$ of the the Lee-Yang-Huang correction caused by quantum depletion of the condensate. This favorable comparison with the exact results available gives insight into the processes that dominate beyond MF theory.

The Hamiltonian density for the system is given by

$$
\begin{aligned}
H= & \bar{\psi}_{\sigma}(x)\left[\frac{-\nabla^{2}}{2 m_{\sigma}}-\mu_{\sigma}\right] \psi_{\sigma}(x) \\
& -g \bar{\psi}_{\uparrow}(x) \bar{\psi}_{\downarrow}(x) \psi_{\downarrow}(x) \psi_{\uparrow}(x),
\end{aligned}
$$

where we use "spin" $\sigma=\uparrow, \downarrow$ to label the two fermion species with masses $m_{\uparrow} \geqslant m_{\downarrow}$. The short-range attraction ${ }^{1}$ with ultraviolet cutoff $\Lambda$ has a strength $g(\Lambda)$ that is related to the $s$-wave scattering length $a_{s}$ via [3] $m / 4 \pi a_{s}=-1 / g(\Lambda)+$ $\sum_{|\mathbf{k}|<\Lambda} m / k^{2}$, with $m$ defined in Eq. (2). We set $\hbar=1$ and work in a box of unit volume.

We have two chemical potentials $\mu_{\sigma}$ to obtain the densities $n_{\sigma}=n / 2$, even in the unpolarized case; see the following. It is convenient to use $\mu=\left(\mu_{\downarrow}+\mu_{\uparrow}\right) / 2$ and $h=\left(\mu_{\downarrow}-\mu_{\uparrow}\right) / 2$ in place of the $\mu_{\sigma}$ and, also, to define the reduced mass $m / 2$ and $0 \leqslant \gamma<1$ given by

$$
\frac{m}{2}=\frac{m_{\uparrow} m_{\downarrow}}{m_{\uparrow}+m_{\downarrow}} \quad \text { and } \quad \gamma=\frac{m_{\uparrow}-m_{\downarrow}}{m_{\uparrow}+m_{\downarrow}} .
$$

We briefly describe our formalism [7] to introduce notation and highlight the changes arising from $m_{\uparrow} \neq m_{\downarrow}$. The partition function $Z\left(T, \mu_{\sigma}\right)$ is written as a functional integral over Grassmann fields. Introducing a Hubbard-Stratonovich field $\Delta(x)$ which couples to $\bar{\psi}_{\uparrow}(x) \bar{\psi}_{\downarrow}(x)$ and integrating out the

\footnotetext{
${ }^{1}$ This corresponds to the wide resonance limit; see Ref. [13].
} 


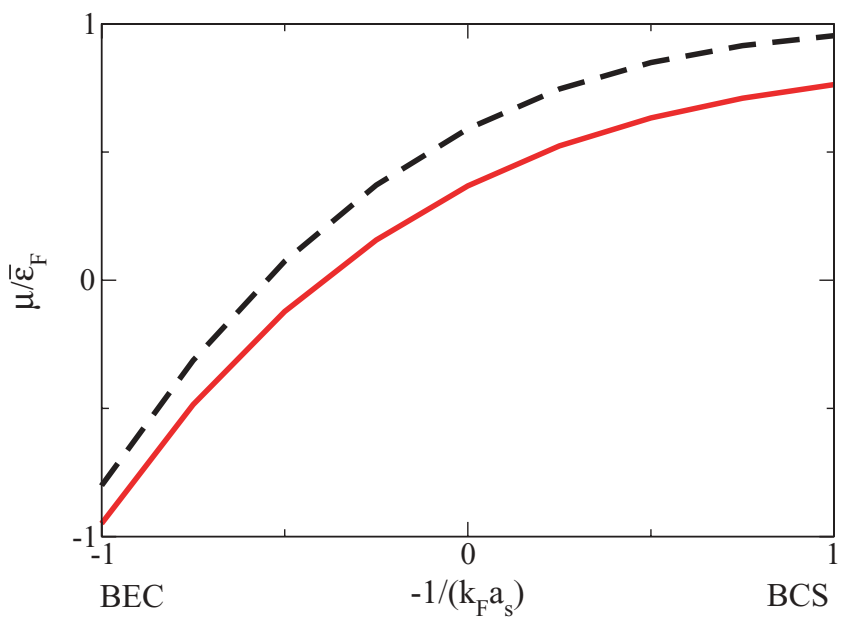

FIG. 1. (Color online) Chemical potential $\mu$ normalized by $\bar{\epsilon}_{F}=\left(\epsilon_{F \uparrow}+\epsilon_{F \downarrow}\right) / 2$ as a function of the interaction $-1 /\left(k_{F} a_{s}\right)$, with $m_{\uparrow} / m_{\downarrow}=6.67$, for ${ }^{40} \mathrm{~K}$ and ${ }^{6} \mathrm{Li}$. The solid (red) line includes Gaussian fluctuations, while the dashed (black) line is the mean field prediction (independent of $m_{\uparrow} / m_{\downarrow}$ ).

fermions, we obtain $Z=\int D \Delta D \Delta^{*} e^{-S}$, with the action $S=\int d x\left\{|\Delta(x)|^{2} / g-\operatorname{Tr} \ln \mathbf{G}^{-1}[\Delta(x)]\right\}$. The integration is over $x=(\mathbf{x}, \tau)$, where the imaginary time $0 \leqslant \tau \leqslant \beta=1 / T$. The inverse Green's function is

$$
\begin{aligned}
\mathbf{G}^{-1}= & {\left[\left(-\partial_{\tau}-h-\gamma \nabla^{2} / 2 m\right) \hat{1}\right.} \\
& \left.+\left(\nabla^{2} / 2 m+\mu\right) \hat{\tau}_{3}+\Delta \hat{\tau}^{+}+\Delta^{*} \hat{\tau}^{-}\right] \delta\left(x-x^{\prime}\right),
\end{aligned}
$$

and the trace in the action $S$ acts on Nambu-Gorkov space.

MF theory corresponds to a uniform, static saddle point $\Delta_{0}$ for $Z$. We find the gap equation $\delta S_{0} / \delta \Delta_{0}=0$, with $S_{0}=$

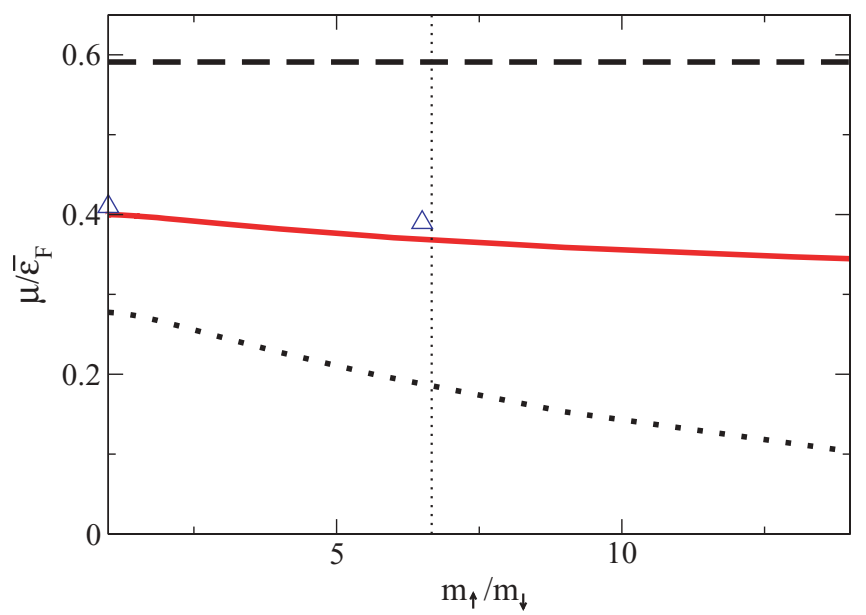

FIG. 2. (Color online) Chemical potential $\mu$ at unitarity as a function of mass ratio. At unitarity, the ground state energy $E$ and $\mu$ are related by universality: $\mu / \bar{\epsilon}_{F}=E /\left(3 N \bar{\epsilon}_{F} / 5\right)=(1+\beta)$, where $\bar{\epsilon}_{F}=\left(\epsilon_{F \uparrow}+\epsilon_{F \downarrow}\right) / 2$. Our calculation including Gaussian fluctuations is the solid (red) line. The vertical line marks $m_{\uparrow} / m_{\downarrow}=6.67$ for a $\mathrm{K}$-Li mixture. For comparison, the two triangles are quantum Monte Carlo results [11], the upper, dashed line is the mean field result, and the lower, dotted line is the result of the $1 / \mathcal{N}$ expansion.

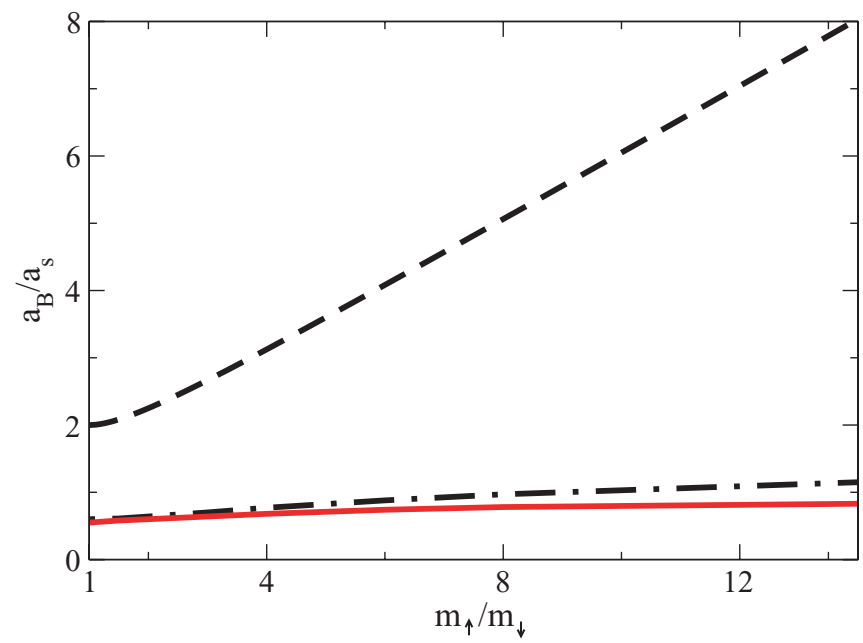

FIG. 3. (Color online) Molecular scattering length in the BEC limit as a function of mass ratio for different calculations. Our calculation including Gaussian fluctuations is the solid (red) line; the exact four-body calculation [12] is shown by the dot-dashed line. The mean field value, corresponding to the Born approximation for dimer scattering, is the upper, dashed line.

$$
\begin{aligned}
S\left[\Delta_{0}\right]= & \beta \Delta_{0}^{2} / g-\sum_{\mathbf{k}, i k_{n}} \operatorname{Tr} \ln \mathbf{G}_{0}^{-1}(k) . \text { Here } \\
& \mathbf{G}_{0}^{-1}(k)=\left(i k_{n}-h+\gamma \epsilon\right) \hat{1}-\xi \hat{\tau}_{3}+\Delta_{0} \hat{\tau}_{1},
\end{aligned}
$$

with fermionic Matsubara frequencies $i k_{n}=i(2 n+1) \pi / \beta$. To simplify notation we omit $\mathbf{k}$ labels on all energies and write them as $\epsilon=|\mathbf{k}|^{2} / 2 m$ and $\xi=\epsilon-\mu$.

The fermionic excitation energies, determined from the poles of $\mathbf{G}_{0}\left(\mathbf{k}, \omega+i 0^{+}\right)$, are

$$
E_{1,2}=E \mp(\gamma \epsilon-h) \text { with } E=\left(\xi^{2}+\Delta_{0}^{2}\right)^{1 / 2} .
$$

The thermodynamic potential $\Omega=-\ln Z / \beta$ at $T=0$ in MF theory is given by

$$
\begin{aligned}
\Omega_{0} & =S_{0} / \beta \\
& =\Delta_{0}^{2} / g+\sum_{\mathbf{k}}\left[\xi-E+E_{1} \Theta\left(-E_{1}\right)+E_{2} \Theta\left(-E_{2}\right)\right] .
\end{aligned}
$$

Precisely at $T=0$, given $\mu, \Delta_{0}$, and $\gamma$, there is a range of $h$ values for which we obtain an unpolarized solution $n_{\uparrow}=n_{\downarrow}$ with $E_{1,2}>0$. This range is $\max (\gamma \epsilon-E)=A^{(-)} \leqslant$ $h \leqslant A^{(+)}=\min (\gamma \epsilon+E)$, where the $\max / \min$ are for $\epsilon \geqslant 0$. It is easy to see that $A^{( \pm)}=\gamma \mu \pm \sqrt{1-\gamma^{2}} \Delta_{0}$ for $\mu / \Delta_{0} \geqslant$ $\pm \Gamma$, while $A^{( \pm)}= \pm \sqrt{\mu^{2}+\Delta_{0}^{2}}$ for $\mu / \Delta_{0}< \pm \Gamma$, where $\Gamma \equiv$ $\gamma / \sqrt{1-\gamma^{2}}$. In the limit $T \rightarrow 0$ there is a unique solution, ${ }^{2}$ $h=\left[A^{(+)}+A^{(-)}\right] / 2$; deviations away from this value lead

\footnotetext{
${ }^{2}$ The $\quad$ MF $\quad$ result $\quad \sum_{\mathbf{k}}\left[\tanh \left(\beta E_{1} / 2\right)-\tanh \left(\beta E_{2} / 2\right)\right]=0$ can be rewritten as $\exp (2 \beta h)=I^{(-)} / I^{(+)}$, where $I^{( \pm)}=$ $\int_{0}^{\infty} d \epsilon \sqrt{\epsilon} \exp [-\beta(E \pm \gamma \epsilon)]$. We find $h=\left[A^{(+)}+A^{(-)}\right] / 2$ by evaluating $I^{( \pm)} \approx \exp \left[\mp \beta A^{( \pm)}\right]$for $T \rightarrow 0$, since the integral is dominated by the region where the exponent reaches its maximum. At the Gaussian level $\mathbf{M}$ is independent of $h$; thus $\partial \Omega / \partial h=\partial \Omega_{0} / \partial h$ and the MF result for $h$ remains valid.
} 
to an exponentially small polarization at low $T$ within the interval.

The expression for $S_{0}$ simplifies greatly with $E_{1,2}>0$ and we obtain the gap equation,

$$
-m / 2 \pi a_{s}=\sum_{\mathbf{k}}\left(E^{-1}-\epsilon^{-1}\right),
$$

and MF number equation,

$$
n=-\partial \Omega_{0} / \partial \mu=\sum_{\mathbf{k}}(1-\xi / E) .
$$

Thus the properties of the $m_{\uparrow} \neq m_{\downarrow}$ system at the MF level are completely equivalent to those of an equal mass system with chemical potential $\mu=\left(\mu_{\downarrow}+\mu_{\uparrow}\right) / 2$ and $m$ twice the reduced mass, Eq. (2). This was noted in Ref. [8]; here we go beyond that MF analysis by including the effect of Gaussian fluctuations on the equation of state.

We write $\Delta(x)=\Delta_{0}+\eta(x)$, where the $\eta$ are $(\mathbf{x}, \tau)$ dependent fluctuations about the saddle point $\Delta_{0}$. Fourier transforming to momentum $\mathbf{q}$ and bosonic frequencies $i q_{\ell}=i 2 \pi \ell / \beta$ and expanding to quadratic order in $\eta$, we get $S \simeq S_{0}+S_{g}$, where $S_{g}=\frac{1}{2} \sum_{\mathbf{q}, i q_{l}} \eta^{\dagger} \mathbf{M} \eta$ with $\eta^{\dagger}=\left[\eta^{*}(q), \eta(-q)\right]$. The inverse fluctuation propagator $\mathbf{M}$ is given by

$$
\begin{aligned}
\mathbf{M}_{11}(q) & =\frac{1}{g}+\beta^{-1} \sum_{\mathbf{k}, i k_{n}} \mathbf{G}_{22}^{0}(k) \mathbf{G}_{11}^{0}(k+q) \\
& =\frac{1}{g}+\sum_{\mathbf{k}}\left[\frac{u^{2} u^{\prime 2}}{i q_{\ell}-E_{1}-E_{2}^{\prime}}-\frac{v^{2} v^{\prime 2}}{i q_{\ell}+E_{1}+E_{2}^{\prime}}\right], \\
\mathbf{M}_{12}(q) & =\mathbf{M}_{21}(q)=\beta^{-1} \sum_{\mathbf{k}, i k_{n}} \mathbf{G}_{12}^{0}(k) \mathbf{G}_{12}^{0}(k+q) \\
& =\sum_{\mathbf{k}}\left[\frac{u v u^{\prime} v^{\prime}}{i q_{\ell}+E_{1}+E_{2}^{\prime}}-\frac{u v u^{\prime} v^{\prime}}{i q_{\ell}-E_{1}-E_{2}^{\prime}}\right],
\end{aligned}
$$

with $\mathbf{M}_{22}(q)=\mathbf{M}_{11}(-q)$, and in both cases the second line is valid at $T=0$. Here we use standard BCS notation, $v^{2}=1-u^{2}=\frac{1}{2}[(1-(\xi / E)]$, together with the abbreviations $u=u_{\mathbf{k}}, u^{\prime}=u_{\mathbf{k}+\mathbf{q}}$, etc. The only difference in $\mathbf{M}_{i j}$ from the equal-mass case is the presence of $E_{1}+E_{2}^{\prime}=E+E^{\prime}-$ $\gamma\left(\epsilon^{\prime}-\epsilon\right)$. Thus, even at the Gaussian level, $h$ does not enter the calculation as long as the system is unpolarized.

The partition function is now approximated by $Z \simeq$ $\exp \left(-S_{0}\right) \int D \eta D \eta^{\dagger} \exp \left(-S_{g}\right)$. As explained in Refs. [7] and [14] the saddle-point equation still retains its earlier form, $\delta S_{0} / \delta \Delta_{0}=0$, but the thermodynamic potential $\Omega$ at $T=0$ now includes the contributions of the energy of zero-point motion of the collective excitations [the Goldstone mode of the broken U(1) symmetry in the superfluid] and the virtual scattering of gapped fermionic quasiparticles. A careful consideration of convergence factors [7] leads to $\Omega \simeq \Omega_{0}+\Omega_{g}+\ldots$, with

$$
\Omega_{g}=\frac{1}{2 \beta} \sum_{\mathbf{q}, i q_{\ell}} \ln \left[\mathbf{M}_{11}(q) \operatorname{Det} \mathbf{M}(q) / \mathbf{M}_{22}(q)\right] e^{i q_{\ell} 0^{+}} .
$$

The Gaussian contribution leads to a lowering of the ground state energy and a modified equation of state, which we calculate as $n=-\partial \Omega / \partial \mu$ at $T=0$. Given the dependence of $\Delta_{0}$ on $\mu$, we thus find

$$
n=-\partial \Omega_{0} / \partial \mu-\partial \Omega_{g}\left[\mu, \Delta_{0}(\mu)\right] / \partial \mu .
$$

In Fig. 1 we show the average chemical potential $\mu$ as a function of the scattering length $a_{s}$ for the experimentally relevant case of a ${ }^{40} \mathrm{~K}-{ }^{6} \mathrm{Li}$ mixture.

To understand in greater detail how unequal masses alter the results from the well-studied equal mass case, we next concentrate on two regions: unitarity and the BEC limit. It is also useful to compare our results with those of a closely related approach, where the Hamiltonian (1) is generalized to $\mathcal{N}$ flavors of fermions of each species, interacting with an $\operatorname{Sp}(2 \mathcal{N})$ symmetric potential [9]. Then MF theory becomes exact in the $\mathcal{N} \rightarrow \infty$ limit and the Gaussian correction is of order $1 / \mathcal{N}$ so that $\Omega / \mathcal{N}=\Omega_{0}+\left(\Omega_{g} / \mathcal{N}\right)+O\left(1 / \mathcal{N}^{2}\right)$. Thus, we write $\mu=\mu_{0}+\mu_{1} / \mathcal{N}$, where $\mu_{0}$ is the MF value obtained from $n=$ $-\partial \Omega_{0} / \partial \mu$, and $\mu_{1}=-\left(\partial \Omega_{g} / \partial \mu\right) /\left(\partial^{2} \Omega_{0} / \partial \mu^{2}\right)$ evaluated at $\mu_{0}{ }^{3}$ To obtain results relevant to the original Hamiltonian, $\mathcal{N}=1$ is set at the end of the calculation. In contrast, in our Gaussian theory we work directly with the Hamiltonian (1), keeping $\mathcal{N}=1$ from the outset, and calculate corrections to various quantities (like the chemical potential) without assuming that the changes are small.

At unitarity $a_{s}=\infty$ and the interactions have no scale; the chemical potential is the only energy available [15]. The saddle-point condition implies $\mu=0.860 \Delta_{0}$ independent of mass ratio and the thermodynamic potential has the universal scaling form

$$
\Omega(\mu)=\frac{-2}{15 \pi^{2}} \mathcal{F}\left(m_{\uparrow} / m_{\downarrow}\right) \mu^{5 / 2}(2 m)^{3 / 2},
$$

where the function $\mathcal{F}$ is normalized so that $\mathcal{F}(1)=1$ for noninteracting fermions with equal masses.

In the $\mathrm{MF}$ approximation $\mathcal{F}_{0}=2.2032$, independent of $m_{\uparrow} / m_{\downarrow}$, which yields the equation of state $\mu_{0}=\bar{\epsilon}_{F} / \mathcal{F}_{0}^{2 / 3}$, where $\bar{\epsilon}_{F}=k_{F}^{2} /(2 m)=\left(\epsilon_{F \uparrow}+\epsilon_{F \downarrow}\right) / 2$. The Gaussian contribution $\mathcal{F}_{g}$ has a nontrivial, though weak, dependence on the mass ratio obtained by evaluating Eq. (11) numerically. This yields $\mu=\bar{\epsilon}_{F} /\left[\mathcal{F}_{0}+\mathcal{F}_{g}\left(m_{\uparrow} / m_{\downarrow}\right)\right]^{2 / 3}$. This result is plotted by the solid line in Fig. 2, with the dashed line showing the MF result. The dotted line corresponds to the $1 / \mathcal{N}$ result $\mu=\left[1-2 \mathcal{F}_{g}\left(m_{\uparrow} / m_{\downarrow}\right) / 3 \mathcal{N} \mathcal{F}_{0}\right] \mu_{0}$, with $\mathcal{N}=1$. In Fig. 2 we also show the result of a recent quantum Monte Carlo calculation [11] (triangles) which agrees fairly well with our approximation.

Let us next discuss the BEC limit $\left(a_{s} \rightarrow 0^{+}\right)$, where we obtain a condensate of tightly bound diatomic molecules of binding energy $E_{B}=1 /\left(m a_{s}^{2}\right)$, mass $m_{B}=m_{\uparrow}+m_{\downarrow}$ and density $n_{B}=n / 2$. These bosonic dimers are weakly interacting with a (dimer) scattering length $a_{B}$, which we now compute. We find it convenient to define dimensionless variables $x$ and $y$ as $|\mu|=(1-y) /\left(2 m a_{s}^{2}\right)$, with $0 \leqslant y \ll 1$ and $\left(2 m a_{s}^{2} \Delta_{0}\right)^{2}=$ $x \ll 1$ in the BEC limit. We can thus expand to quadratic order $S_{0}(x, y) / \beta \simeq\left[-x y+x^{2} / 16\right] /\left(32 \pi m a_{s}^{5}\right)$. The gap equation $\partial S_{0} / \partial x=0$ then yields $x=8 y$.

At the MF level $\Omega_{0}(\mu)=S_{0}(x=8 y, y) / \beta$ so that the number equation $n=-\left(2 m a_{s}^{2}\right) \partial \Omega_{0} / \partial y$ yields $y=2 \pi n a_{s}^{3}$. We

\footnotetext{
${ }^{3}$ This equation of state is equivalent [14] to that in Ref. [9].
} 
find $\mu \simeq\left[-1 /\left(2 m a_{s}^{2}\right)\right]+\left[\pi n a_{s} / m\right]$, and on identifying the second term with $\mu_{B} / 2=2 \pi n_{B} a_{B} / m_{B}$, we obtain the MF result [8] $a_{B}^{\mathrm{MF}} / a_{s}=\left(m_{\uparrow}+m_{\downarrow}\right) / m$. This corresponds to the Born approximation for dimer scattering and is shown as the dashed line in Fig. 3.

The Gaussian contribution, Eq. (11), in the BEC limit can be written as $\Omega_{g}=-x^{2} g\left(m_{\uparrow} / m_{\downarrow}\right) /\left[512 \pi m a_{s}^{5}\right]$, where the function $g\left(m_{\uparrow} / m_{\downarrow}\right)$ can be numerically calculated by the method of Ref. [7]. Using $x=8 y$, we write $\Omega(\mu)=\Omega_{0}+$ $\Omega_{g}=-(1+g) y^{2} /\left(8 \pi m a_{s}^{5}\right)$. Solving the number equation $n=-\left(2 m a_{s}^{2}\right) \partial \Omega / \partial y$ for $\mu$, we determine the dimer scattering length (as explained previously) to find ${ }^{4} a_{B} / a_{s}=\left(m_{\uparrow}+\right.$ $\left.m_{\downarrow}\right) /\left\{\left[1+g\left(m_{\uparrow} / m_{\downarrow}\right)\right] m\right\}$. This is plotted as the solid line in Fig. 3 and compares very well with the exact result from a four-body calculation of the scattering between dimers [12].

We use our numerical results to find the next correction in the BEC limit, by fitting $\Omega(\mu)$ at small $y$ to to the form $\left(A y^{2}+B y^{5 / 2}+\cdots\right) /\left(m a_{s}^{5}\right)$. We thus find the $T=$ 0 equation of state of the Lee-Yang-Huang form $\mu_{B}=$ $\left(4 \pi n_{B} a_{B} / m_{B}\right)\left[1+32 C\left(n_{B} a_{B}\right)^{3 / 2} /(3 \sqrt{\pi})+\cdots\right]$ arising from the quantum depletion of the condensate. From our numerics we find $C=0.90 \pm 0.05$ over the mass ratio range $1 \leqslant$ $m_{\uparrow} / m_{\downarrow} \leqslant 13.6$. The exact result [16] for the dilute Bose gas is $C=1$.

Finally, we analyze the effect of a harmonic trap within the local density approximation (LDA); for simplicity we discuss spherical traps. The two species will in general see different trapping potentials with frequencies $\omega_{\sigma}$, so that within LDA $\mu_{\sigma}(r)=\left(\overline{\mu_{\sigma}}-m_{\sigma} \omega_{\sigma}^{2} r^{2}\right) / 2$. Let us focus on unitarity, where

\footnotetext{
${ }^{4}$ We show elsewhere [14] that the $1 / \mathcal{N}$ result for the dimer scattering length at $\mathcal{N}=1$ is the same as the Gaussian result reported here.
}

$\Delta_{0}(r) / \mu(r)=1.162 \equiv \delta$ for all $r$. To ensure zero polarization at $T=0$ we must satisfy $h(r) / \mu(r)=K$. Using the results derived here, Eq. (3), $K=\gamma$ for $\gamma / \sqrt{1-\gamma^{2}}<1 / \delta$, corresponding to $m_{\uparrow} / m_{\downarrow}<4.74$, while $K=\left[\gamma-\delta \sqrt{1-\gamma^{2}}+\sqrt{1+\delta^{2}}\right] / 2$ for $m_{\uparrow} / m_{\downarrow}>4.74$. То maintain this ratio of $h / \mu$ we must choose

$$
\frac{\omega_{\uparrow}^{2}}{\omega_{\downarrow}^{2}}=\frac{m_{\downarrow}(1-K)}{m_{\uparrow}(1+K)} .
$$

Thus, $\omega_{\uparrow} / \omega_{\downarrow}=m_{\downarrow} / m_{\uparrow}$ for $m_{\uparrow} / m_{\downarrow}<4.74$, while for an $\mathrm{Li} / \mathrm{K}$ mixture, where $K=0.745$, we get $\omega_{\uparrow} / \omega_{\downarrow} \simeq 0.148$. Deviations from this frequency ratio would lead to regions of spin imbalance in the trap.

We conclude with a brief discussion of some general issues. First, note that mass imbalance does not impact pairing of "time-reversed" states. For the unpolarized case the Fermi spheres match in $\mathbf{k}$ space even if the $\mu$ values are different. Unlike in many condensed matter systems, where time-reversal breaking perturbations [17] flip the spin of the fermions, atomic species retain their identity ("spin") in collisions. However, effects beyond pairing (which is what we have focused on) may well destabilize the system. In the BEC limit, it is known that for $m_{\uparrow} / m_{\downarrow}>13.6$ the presence of Efimov states makes the Feshbach molecules unstable to collapse by three-body recombination [12]. Finally, we may ask why our approach seems to work so well compared with available exact results (in the stable mass ratio regime). The answer seems to lie in the fact that in a homogeneous dilute gas (with $k_{F}^{-1} \gg$ range of potential), particle-hole channel contributions are negligible compared to the particle-particle channel physics captured in our approach.

We gratefully acknowledge support from ARO W911NF08-1-0338 and NSF-DMR 0706203.
[1] A. J. Leggett, in Modern Trends in the Theory of Condensed Matter, edited by A. Pekalski and R. Przystawa (SpringerVerlag, Berlin, 1980).

[2] P. Noziéres and S. Schmitt-Rink, J. Low Temp. Phys. 59, 195 (1985).

[3] C. A. R. Sá de Melo, M. Randeria, and J. R. Engelbrecht, Phys. Rev. Lett. 71, 3202 (1993); J. R. Engelbrecht, M. Randeria, and C. A. R. Sá de Melo, Phys. Rev. B 55, 15153 (1997).

[4] C. A. Regal, M. Greiner, and D. S. Jin, Phys. Rev. Lett. 92, 040403 (2004); M. W. Zwierlein, C. A. Stan, C. H. Schunck, S. M. F. Raupach, A. J. Kerman, and W. Ketterle, ibid. 92, 120403 (2004); K. M. O’Hara, S. L. Hemmer, M. E. Gehm, S. R. Granade, and J. E. Thomas, Science 298, 2179 (2002); M. Bartenstein, A. Altmeyer, S. Riedl, S. Jochim, C. Chin, J. H. Denschlag, and R. Grimm, Phys. Rev. Lett. 92, 120401 (2004); T. Bourdel, L. Khaykovich, J. Cubizolles, J. Zhang, F. Chevy, M. Teichmann, L. Tarruell, S. J. J. M. F. Kokkelmans, and C. Salomon, ibid. 93, 050401 (2004); J. Kinast, A. Turlapov, J. Thomas, Q. Chen, J. Stajic, and K. Levin, Science 307, 1296 (2005).
[5] E. Wille, F. M. Spiegelhalder, G. Kerner, D. Naik, A. Trenkwalder, G. Hendl, F. Schreck, R. Grimm, T. G. Tiecke, J. T. M. Walraven, S. J. J. M. F. Kokkelmans, E. Tiesinga, and P. S. Julienne, Phys. Rev. Lett. 100, 053201 (2008).

[6] M. Alford, A. Schmitt, K. Rajagopal, and T. Schafer, Rev. Mod. Phys. 80, 1455 (2008).

[7] R. B. Diener, R. Sensarma, and M. Randeria, Phys. Rev. A 77, 023626 (2008).

[8] M. Iskin and C. Sa de Melo, Phys. Rev. A 76, 013601 (2007).

[9] For the equal mass case, see: P. Nikolic and S. Sachdev, Phys. Rev. A 75, 033608 (2007); M. Y. Veillette, D. E. Sheehy, and L. Radzihovsky, ibid. 75, 043614 (2007).

[10] G. E. Astrakharchik, J. Boronat, J. Casulleras, and S. Giorgini, Phys. Rev. Lett. 93, 200404 (2004).

[11] A. Gezerlis, S. Gandolfi, K. E. Schmidt, and J. Carlson, Phys. Rev. Lett. 103, 060403 (2009).

[12] D. S. Petrov, C. Salomon, and G. V. Shlyapnikov, J. Phys. B 38, S645 (2005).

[13] R. B. Diener and T.-L. Ho, e-print arXiv:cond-mat/0405174 (2004). 
[14] R. B. Diener, R. Sensarma, and M. Randeria (unpublished).

[15] G. A. Baker, Int. J. Mod. Phys. B 15, 1314 (2001); H. Heiselberg, Phys. Rev. A 63, 043606 (2001); T. L. Ho, Phys. Rev. Lett. 92, 090402 (2004).
[16] T. D. Lee and C. N. Yang, Phys. Rev. 105, 1119 (1957).

[17] K. Maki, in Superconductivity, Vol. 2, edited by R. D. Parks (Dekker, New York, 1969). 